\title{
Single ectopic ureteral orifice with bilateral duplicated renal collecting systems in an adult girl: Diagnosis by magnetic resonance urography
}

\author{
Min Tang, MD; $;^{*}$ Quanrongzi Wang, MD; ${ }^{\dagger}$ Bianjiang Liu, MD; Jie Li, MD;* Qiang Lu, MD; Ninghong Song, MD;* \\ Zengjun Wang, MD; ${ }^{*}$ Wei Zhang, $M D^{*}$
}

*State Key Laboratory of Reproductive Medicine, Department of Urology, The First Affiliated Hospital of Naniing Medical University, Naniing, China; 'Department of Radiology, The First Affiliated Hospital of Naniing Medical University, Nanijing, China

Cite as: Can Urol Assoc J 2015;9(7-8):E554-8. http://dx.doi.org/10.5489/cuaj.2886 Published online August 10, 2015.

\section{Abstract}

Renal duplication accompanied by ureteral ectopia is an uncommon urinary congenital abnormality. We report the case of a 21-year-old girl who suffered from lifelong continuous urinary leakage. She was finally diagnosed with bilateral duplicated collecting systems complicated with right ectopic ureteral orifice - an extremely rare case. The patient underwent ureteric re-implantation for the ectopic side, and her urinary incontinence ceased soon thereafter. In this case, traditional imaging failed to show the exact insertion of an ectopic ureter. However, magnetic resonance urography combined with retrograde intubation radiography successfully depicted the point of ureteric insertion, which may make the diagnostic process accurate and efficient.

\section{Introduction}

Renal duplication accompanied by ureteral ectopia is a rare urinary congenital abnormality, which is more common in girls. Common sites of ectopic ureteral insertion include below the external urethral sphincter, such as distal urethra, the vagina, uterus or vestibulum. All of these can result in lifelong urinary incontinence if not treated. ${ }^{1}$

When the upper pole moieties are small or dysplastic, diagnosis can be subtle and the condition can go unrecognized by routine methods. ${ }^{2}$ Successful confirmation of the ecotopic ureter openings preoperatively also remains a diagnostic challenge. We report a rare case in which, although conventional imaging demonstrated bilateral duplicated renal collecting systems, it failed to depict the exact site of insertion of the right ectopic ureter. The patient was successfully diagnosed by a magnetic resonance urography (MRU) and retrograde intubation radiography.

\section{Case report}

A 21-year-old girl presented with lifelong continuous urinary leakage from the perineum. She had normal divided urination, but no presentations of urinary infection or lower back pain. She had an unremarkable medical, surgical, and family history. On admission, her physical examination and laboratory data were also unremarkable.

The ultrasound showed a roughly normal right kidney and a duplex left kidney without hydronephrosis (not shown). Intravenous pyelogram (IVP) demonstrated a duplicated collecting system on the left and a suspicious shadow which demonstrated probable right upper pole moiety with its proximal ureter (Fig. 1). A computed tomography (CT) scan was then performed, which revealed bilateral duplex collecting systems in the coronal plane and dilated ureter extending below bladder on the right in axial plane (Fig. 2). A cystoscopy demonstrated normal bilateral ureteral orifices and a retrograde pyelography was performed. The results showed left duplicated kidney (Fig. 3, part A) and a fine developing degree of right lower pole moiety (Fig. 3, part B), but the right upper pole moiety was not seen. Then, methylene blue was poured into the bladder. No fluid drained out from the vaginal orifice. Nevertheless, a little clear liquid discharged from a needle eye-like opening between urethral and vaginal orifice (Fig. 4). Ureteral catheter was then inserted into the opening and upward intubated for about $5 \mathrm{~cm}$; a retrograde urography revealed a dilated, tortuous distal ureteral shadow on the right (Fig. 5).

MRU was finally performed and showed bilateral duplicated collecting systems. The upper pole moiety on the right 


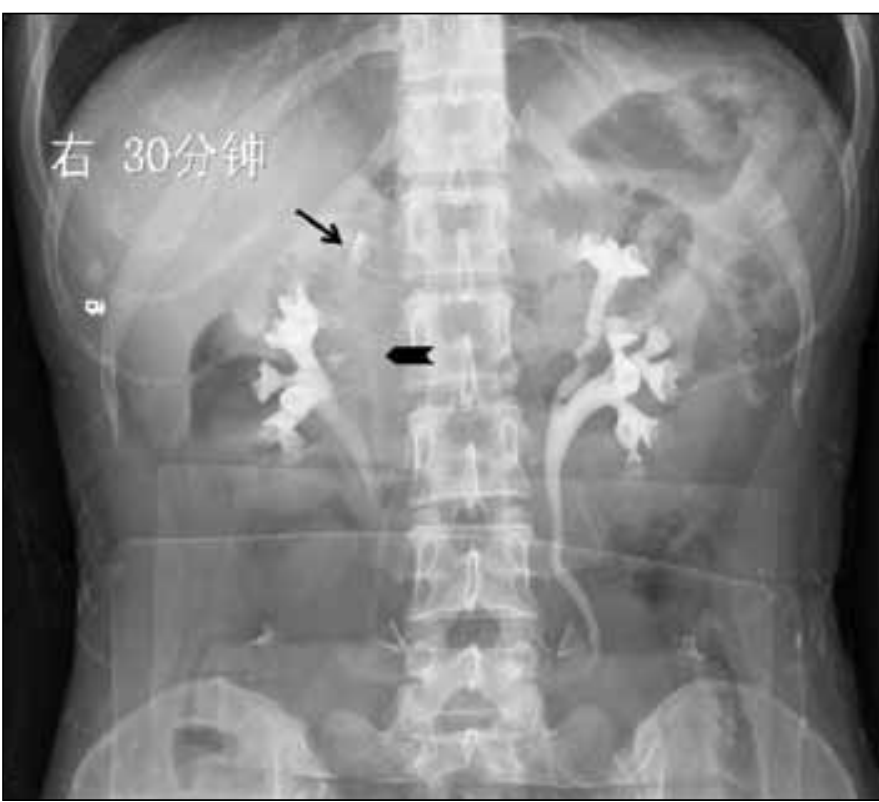

Fig. 1. The intravenous pyelography (IVP) demonstrated a duplicated collecting system on the left, and the upper pole ureter affluxed into the lower pole collecting system. There was also a suspicious shadow which demonstrated probable right upper pole moiety (arrow) with its proximal ureter (arrowhead).

was described as a small soft tissue density shadow above the upper pole of the right kidney. There was no clear distinction between the right lower pole moiety and the upper one. Upon lateral view, the heavily T2 weighted image demonstrated left duplicated renal collecting systems and dilated, tortuous distal ureter on the right hydroureter (Fig. 6). The patient then underwent ureteric re-implantation for the ectopic side; she tolerated the procedure well. Followup revealed complete elimination of the continuous urinary incontinence.
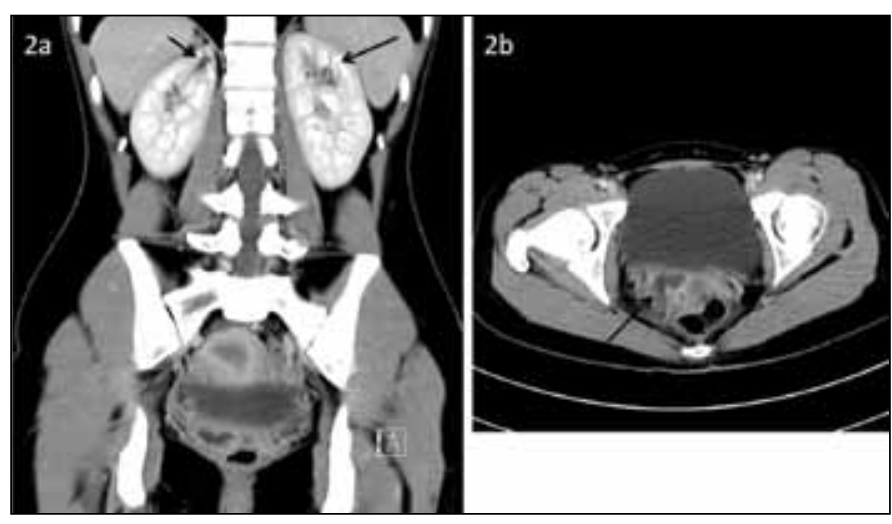

Fig. 2. A: Coronal computed tomography (CT) scan demonstrated bilateral duplicated renal collecting systems (arrows). B: A CT image obtained at a lower level demonstrates the dilated ureter extending below bladder.

\section{Discussion}

Duplex renal collecting system is a common congenital anomaly in the pediatric population, but ectopic ureters are rare, with an incidence between 1 in 2000 and 1 in 4000 found in autopsies in children. Their incidence is at least 4 times higher in females than in males, and about $80 \%$ of patients have associated duplex kidneys. ${ }^{3}$ Bilateral duplicated kidneys accompanied by a single ectopic ureteral orifice are extremely rare.

Dribbling in otherwise normal girls suggests a duplicated collecting system whose upper pole ureter drains ectopically. ${ }^{4}$ Our case presented with classic symptoms. Traditionally, the diagnosis of duplicated renal collecting system and delineation of ectopic ureter orifices are made with ultrasound, IVP, retrograde pyelography, and cystoscopy. ${ }^{5}$ In some situations, an accurate diagnosis may be difficult with these conventional imaging techniques.

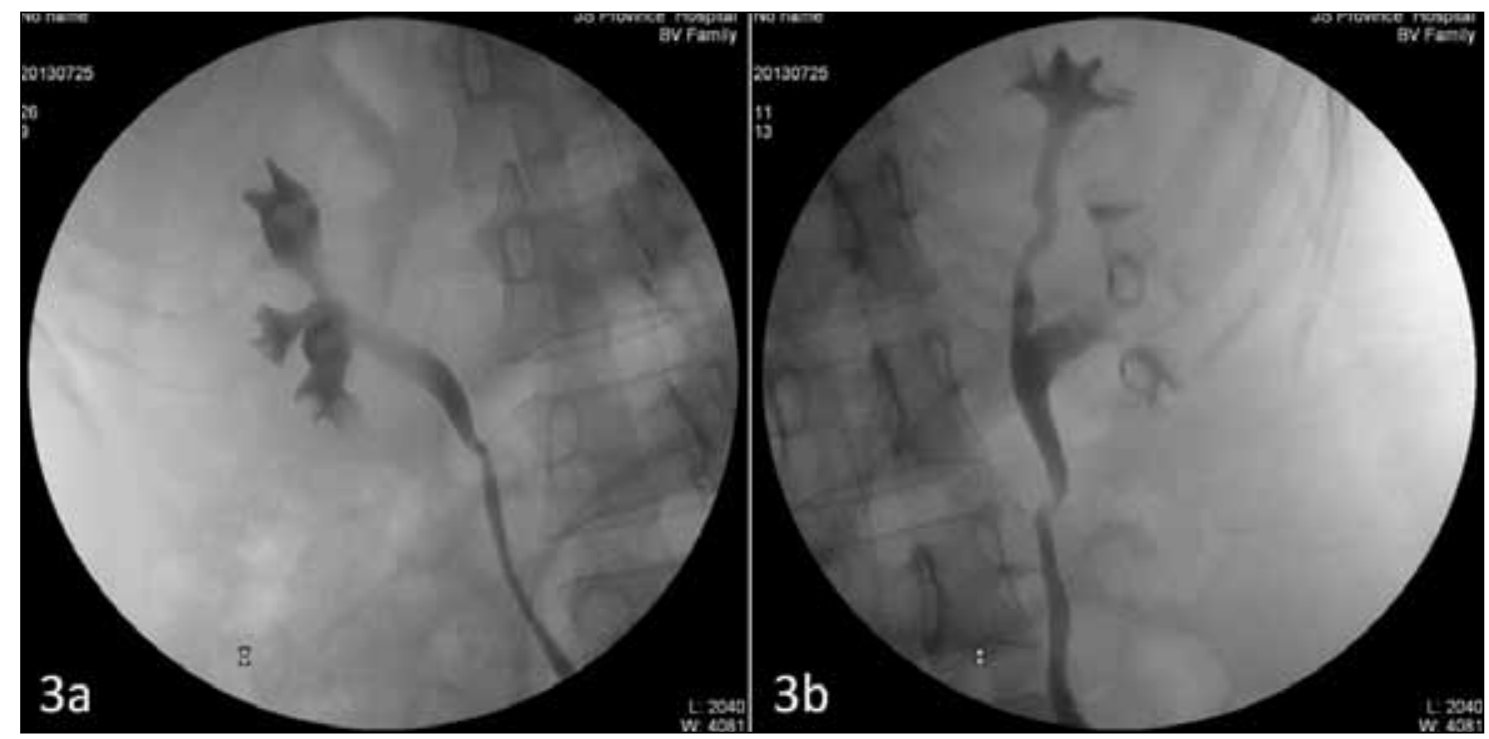

Fig. 3. The retrograde pyelography showed a fine developing degree of right lower pole moiety (A) and left duplicated renal collecting systems (B). 


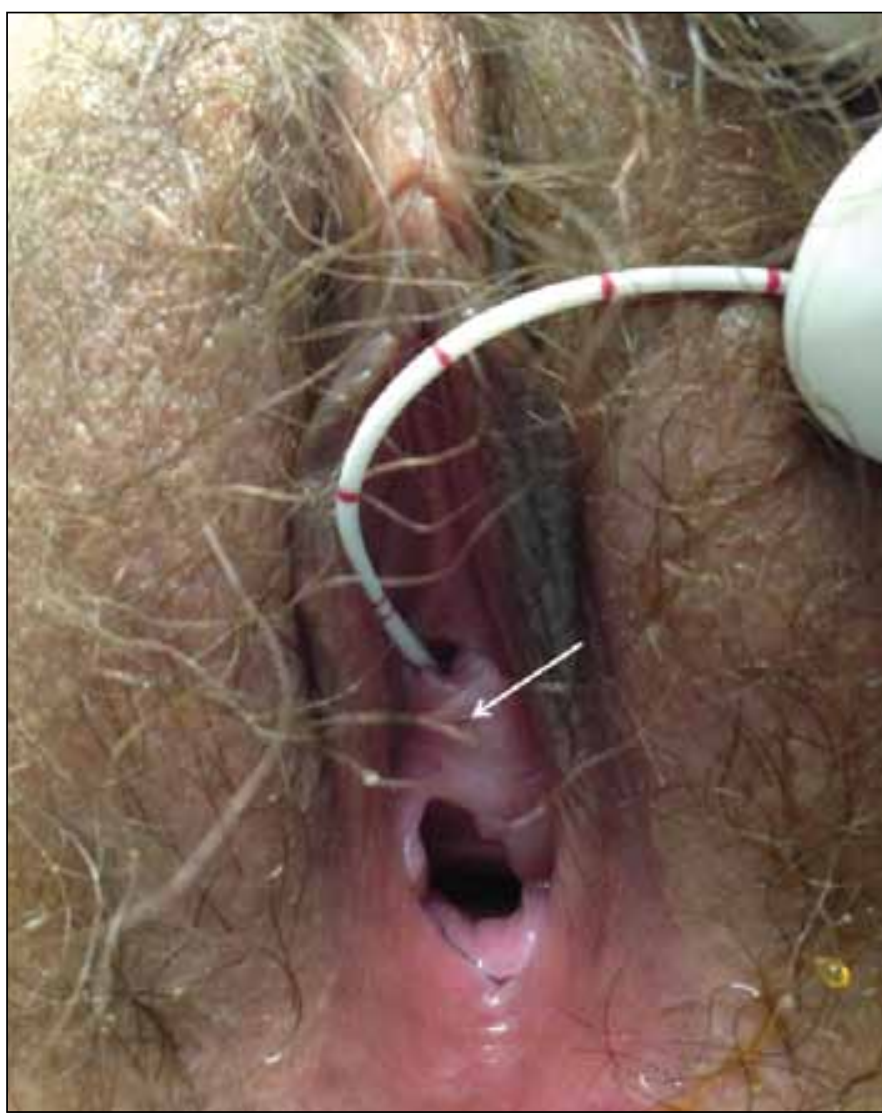

Fig. 4. A needle eye-like opening between urethral and vaginal orifice was found during physical examination (arrow).

Ultrasound is helpful in the diagnosis. In this case, the upper pole moiety of the right duplicated kidney was small and dysplastic and the ultrasound failed to demonstrate duplex renal collecting system. Due to the overlying bowel gas, it would be confusing to the sonographer to confirm the tortuous route of the dilated ureter and depict its entirety.

Usually IVP clearly reveals the full ureter alignment. In this case, the patient received double dose IVP. After 30 minutes of radiography, we saw a blurring and suspicious shadow, suggesting probable right upper pole moiety with its proximal ureter. But the entirety of the right ectopic ureter, as well as the possible ectopic orifice area, could not be seen, which probably indicates a dysplastic and poorly functioning segment. This may have impeded the detection of the segment by IVP despite delayed films. ${ }^{6}$ On this basis, further examinations were needed to get an accurate diagnosis.

Although CT scan revealed bilateral duplicated kidneys and a dilated ureter extending below the bladder on the right, it failed to show the exact insertion of the upper pole ureteral orifice. The possible reason might be low contrast resolution for deep pelvic structures and imaging in the axial plane which could not accurately depict the craniocaudally oriented insertion.

Due to the rarity of a duplicated kidney with incomplete double ureters and the number of ureteric openings during cystoscopy, we were able to confirm the side of the lesion. In our patient, the cystoscopy showed normal bilateral ureteral orifices, but the ectopic ureteral orifice could not be identified. The retrograde pyelography demonstrated duplicated renal collecting systems on the left and a fine developing degree of right lower pole moiety; it did not show the upper pole moiety on the right, which suggested that the ectopic ureter was on the right side and the ectopic orifice might be inserted extravesically.

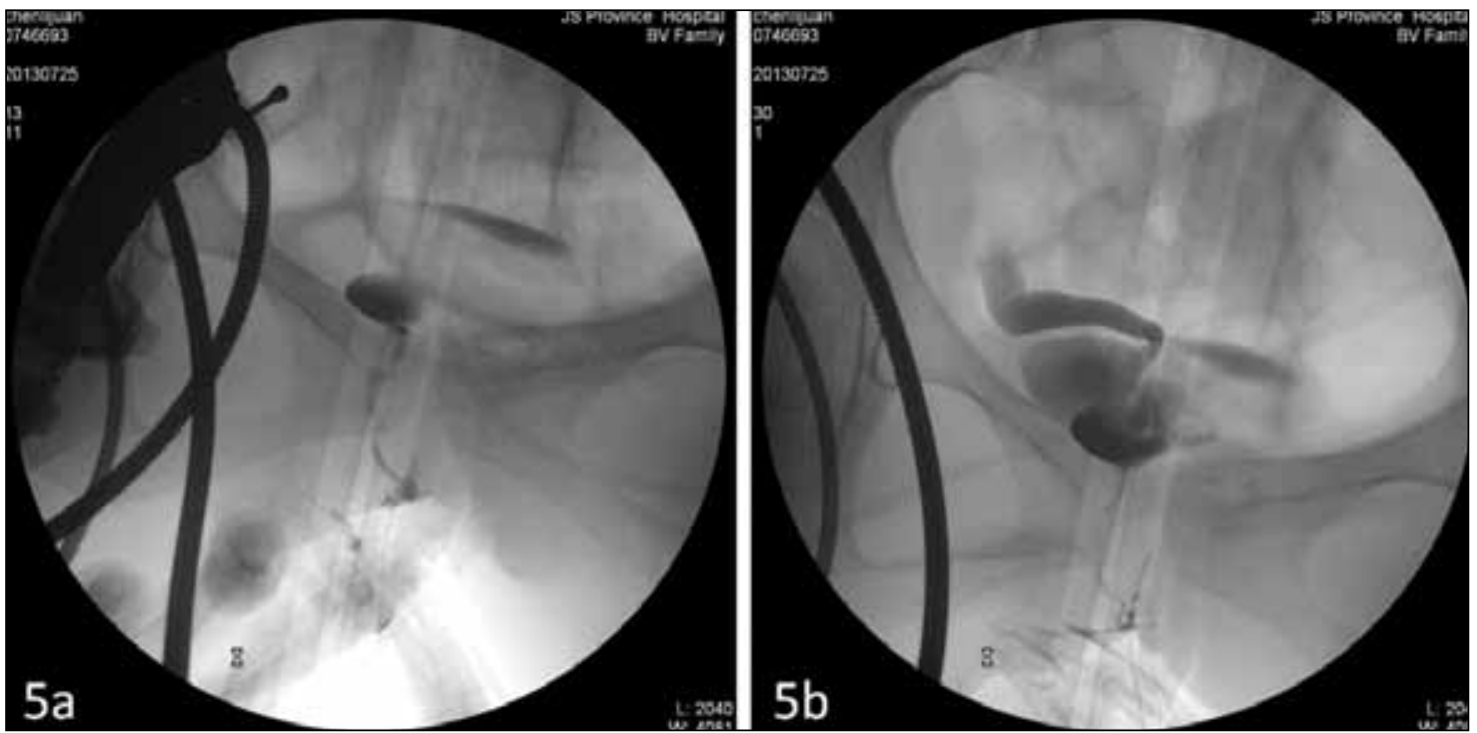

Fig. 5. Retrograde intubation radiography was performed through the suspicious needle eye-like opening. A: Imaging when contrast agent was just injected. B: A dilated, tortuous distal ureteral shadow on the right developed. 

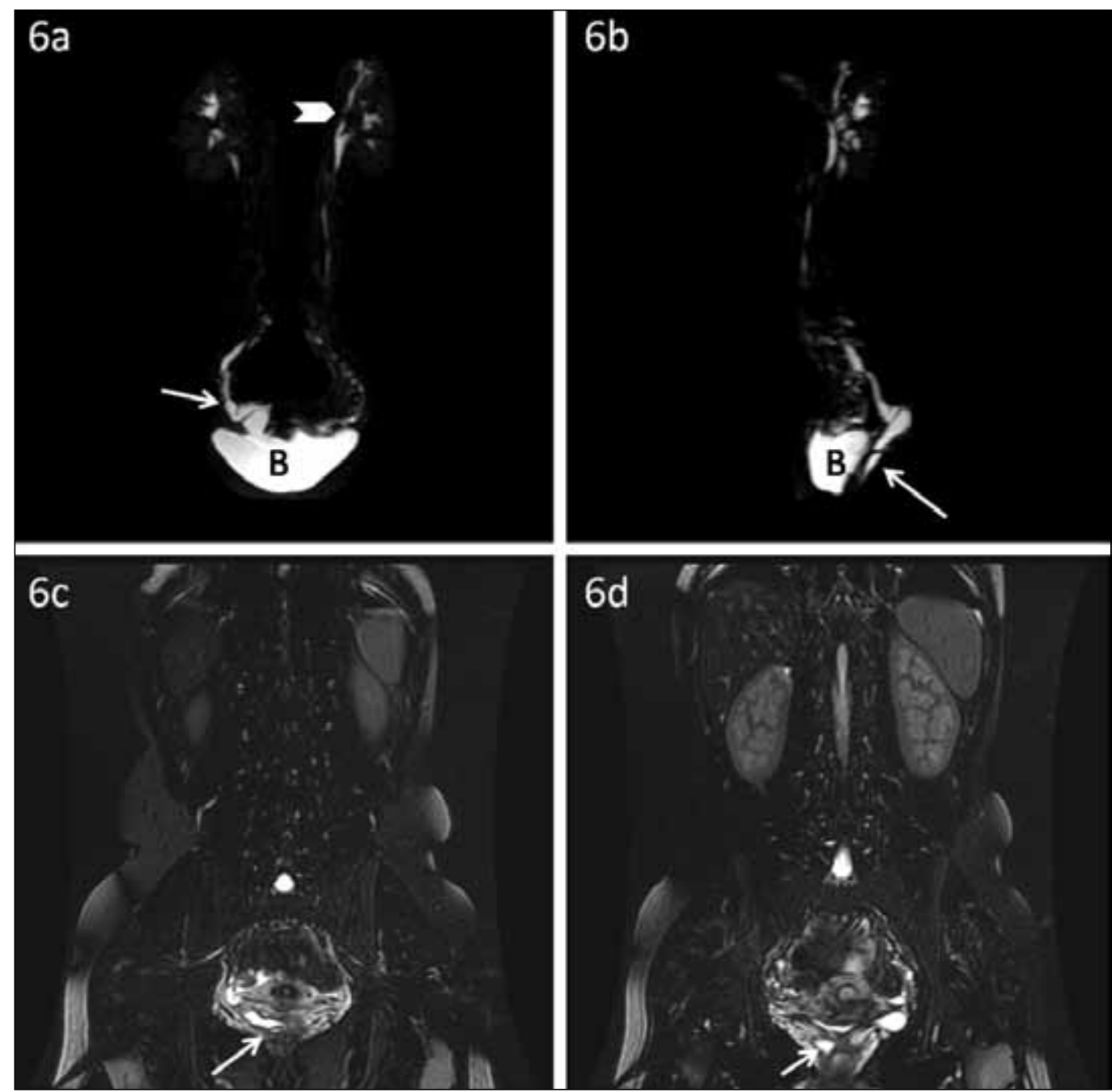

Fig. 6. A: Heavily T2 weighted image demonstrates left duplicated renal collecting systems (arrowhead) and dilated, tortuous distal ureter on the right (arrow). B=bladder. B: Lateral view of the heavily T2 weighted image demonstrates dilated portion of the right hydroureter tracking to the inferior margin of the bladder (arrow). C: Coronal moderately T2 weighted image demonstrates dilated, distal ectopic ureter on the right (arrow). D: The ectopic ureter is inserting towards the paravaginal region (arrow).

A common site for ectopic ureteral insertion is the vestibulum, between the urethral and vaginal orifice. The orifice is usually too small and hidden via routine physical examination. In our patient, we saw clear fluid dripping from a needle eye-like opening between the urethral and vaginal orifice. Even so, we still could not determine whether we were looking at the ectopic ureteral orifice. The clear fluid might just be coming from the normal urethral orifice nearby. Therefore, the retrograde intubation radiography was performed and revealed a dilated, tortuous distal ureteral shadow.

Compared with CT scan, MRI has multiplanar capability and provides high-resolution images of deep pelvic structures. ${ }^{6}$ In addition, the use of T2-weighted MR urography is particularly useful in the detection of ectopic insertion of the ureter. ${ }^{7}$ In this case, MRU could demonstrate the prob- able insertion site of the obstructed ectopic ureter, while ultrasound, IVP, CT scan, and cystoscopy all failed.

\section{Conclusion}

We described the rare case of a patient with bilateral duplicated collecting systems accompanied by right ectopic ureteral orifice. The diagnosis of duplicated kidney with ectopic ureter depends on several imaging techniques, including MRU combined with retrograde intubation radiography to evaluate the kidney to the point of distal ureteral insertion accurately and efficiently.

Acknowledgements: This work was supported by the grants from National Science Foundation of China $(81270685 ; 81200467)$ and by A Project Funded by the Priority Academic Program Development of Jiangsu Higher Education Institutions (JX10231802). 
Tang et al.

Competing interests: The authors declare no competing financial or personal interests.

This paper has been peer-reviewed.

\section{References}

1. Corbett ST, Burris MB, Herndon CD. Pediatric robotic-assisted laparoscopic ipsilateral ureteroureterostomy in a duplicated collecting system. J Pediatr Urol 2013;9:1239.el-2. http://dx.doi.org/10.1016/i. ipurol.2013.07.017

2. Carrico C, Lebowitz RL. Incontinence due to an infrasphincteric ectopic ureter: why the delay in diagnosis and what the radiologist can do about it. Pediatr Radiol 1998;28:942-9. http://dx.doi.org/10.1007/ s002470050506

3. Synder HM. Anomalies of ureter. In: Gillenwater JY, Grayhack JT, Howard SS, Duckeft JW (eds). Adult and pediatric urology. Mosby, St. Louis, 1991:1831-62.
4. Jain KA. Ectopic vaginal insertion of an obstructed duplicated ureter in an adult female: Demonstration by magnetic resonance imaging. Clin Imaging 2007;31:54-6. http://dx.doi.org/10.1016/i.dinimag.2006.07.001

5. Berrocal T, López-Pereira P, Arionilla A, et al. Anomalies of the distal ureter, bladder, and urethra in children: Embryologic, radiologic, and pathologic features. Radiographics 2002;22:1139-64. http:// dx.doi.org/10.1148/radiographics.22.5.g02se101139

6. Engin G, Esen T, Rozanes I. MR urography finds of a duplicated ectopic ureter in an adult man. Eur Radiol 2000;10:1253-6. http://dx.doi.org/10.1007/s003300000319

7. Staatz $\mathrm{G}$, Rohrmann D, Nolte-Ernsting $\mathrm{CC}$, et al. Magnetic resonance urography in children: Evaluation of suspected ureteral ectopia in duplex systems. J Urol 2001;166:2346-50. http://dx.doi.org/10.1016/ S0022-5347(05)65586-3

Correspondence: Dr. Bianiiang Liu, State Key Laboratory of Reproductive Medicine, Department of Urology, The First Affiliated Hospital of Nanijng Medical University, Naniing, China; bjliu@nimu.edu.cn 